\title{
Developing a Neural Network potential to investigate interface phenomena in solid-phase epitaxy
}

\author{
Ruggero Lot \\ LAAS-CNRS \\ Université de Toulouse, CNRS \\ Toulouse, France \\ and SISSA \\ Trieste, Italy \\ rlot@laas.fr
}

\author{
Layla Martin-Samos \\ CNR-IOM \\ Istituto Officina dei Materiali \\ Democritos National Simulation Center \\ Trieste, Italy \\ marsamos@iom.cnr.it
}

\author{
Stefano de Gironcoli \\ SISSA and CNR-IOM \\ Trieste, Italy \\ degironc@sissa.it
$L A A S-C N R S$
Université de Toulouse, CNRS
Toulouse, France
anne.hemeryck@laas.fr \\ Anne Hémeryck
}

\begin{abstract}
In this work, we develop a new neural network potential for silicon and perform accurate molecular dynamics simulations of the liquid, amorphous and diamond phases. The potential is tested against several physical properties and the solid phase epitaxy process is simulated.
\end{abstract}

\section{INTRODUCTION}

Silicon based modern technology is facing an ongoing miniaturization challenge because faster and less powerhungry devices are required for all sort of applications, from small and super portable sensors to the development of new supercomputer chips. Industrial fabrication has already moved towards the exploitation of the third dimension as a way to pack chips reaching the astonishing small scale of tens of nanometer per single transistor. At such a small scale a plethora of phenomena that drive the device formation need a more accurate theoretical understanding to allow for more reliable device production.

In the multi-scale modeling approach often adopted to explain macroscopic experimental observations [1], a critical step that drastically impacts the accuracy of the theoretical prediction is the choice of an empirical potential to describe the atomistic scale. Common empirical potentials used in studies of the liquid, amorphous and diamond phases - crucial for the investigation of any step in the formation of a silicon device - have more or less complex functional forms, inspired by intuition and physical insight, with few parameters fitted either to macroscopic properties or accurate calculations [2], [3]. One of the big problems of these potentials is their lack of flexibility, so that if they aim to be general they suffer limited accuracy and can fail to correctly describe the individual phases. On the other hand their great advantage is to be computationally very efficient as compared to more accurate first-principles calculations.

In recent years very flexible machine-learning (ML) potentials, trained on ab-initio data, are emerging as an attempt to get the best of the two worlds [4]-[9]: reaching the accuracy of first principle calculations at the computational cost of an empirical potential. Several different ML techniques are available, with advantages and disadvantages [9]. After sketching the methodology in Sec. II, Sec. III and Sec. IV are dedicated to the creation of the dataset and the testing of the potential. Sec. V simulates the solid phase epitaxy (SPE).

\section{Methodology}

The construction of a neural network potential can follow several approaches [7]. We adopted the method originally proposed by Behler et al. [8] for its generality, that allows the treatment of systems with a varying number of atoms, and gives a good compromise between speed and accuracy. Within this framework, as for most empirical potentials, one makes the assumption that the total energy $E$ of a system can be expressed as a sum over atomic energy contributions:

$$
E=\sum_{i} E_{i} .
$$

where the index $i$ labels the atoms in the unit cell.

Each atomic energy term is expressed as a function of the local environment surrounding a central atom and is realized by a neural networks (NN). Different NNs are used to compute the contribution of different atomic species.

The complete definition of a NN potential requires the specification of i) an encoding for the local atomic environments in the form of a vector that can be used as input to the NN and ii) the specification of the $\mathrm{NN}$ architecture (shape) to process it. Here the input, or descriptor, is implemented adopting the Symmetry Function (SF) concept first proposed in [8] and later extended in [4], [6]. SFs are continuous many-body functions $G\left(\left\{\mathbf{r}_{i}|| \mathbf{r}_{i}-\mathbf{r}_{r e f} \mid<r_{c}\right\}\right)$ of the atomic coordinates $\mathbf{r}_{i}$ within a cutoff distance, $r_{c}$, from a reference atom (situated at $\mathbf{r}_{r e f}$ ) that encode the local information in a translationally, rotationally and permutationally invariant way. The adopted descriptor comprises purely radial SF components, sampling the distance between $r_{\text {min }}=0.5 \AA$ and $r_{c}=4.6 \AA$ over 32 bins, and combined angular-radial terms with 12 angular and 
6 radial bins between 0 and $\pi$ and 1.5 and $4.6 \AA$, respectively. Further hyperparameters were fixed to $\eta=16.0$ in the radial terms and $\xi=50$ and $\eta=6.0$ in the angular-radial part. This results in SFs with a total of 104 (32 radial and $12 \times 6=72$ angular) components.

As for the specific shape of the NN we adopted an all-to-allconnected feed-forward (FFNN) architecture for its flexibility. Formally a $L$-layer FFNN can be defined as:

$$
x_{i}^{(l)}=f^{(l)}\left(\sum_{j}^{\text {nodes }_{l-1}} w_{i, j}^{(l)} x_{j}^{(l-1)}+b_{i}^{(l)}\right), \quad l=1, \ldots, L,
$$

where for each layer, $l$, and each of its nodes, $i$, the output, $x_{i}^{(l)}$, is formed as a linear combination of the previous layer information using layer dependent bias, $b_{i}^{(l)}$, and weights, $w_{i, j}^{(l)}$, and passing this intermediate result through a layer dependent activation function, $f^{(l)}$. The special case $x_{j}^{(0)}$ is the input vector that is in our case the collection of 104 SF components, while $x_{1}^{(L)}$ is the single neuron of the output layer containing the predicted atomic energy contribution. A sketch of the SF generation and of the $\mathrm{NN}$ architecture used can be seen in the left panel of Fig. 1.

In our case we used two hidden layers (hence $L=3$ ) of 256 and 128 nodes with hyperbolic tangent activation functions and a single output node with linear activation. This is at variance with the Gaussian activation previously adopted [4]-[6] and required a precondition of the input that must be transformed from the positive defined SF to a symmetrical distribution around the origin. To obtain this we added a linear, non trainable, layer to the network to perform a precomputed principal components analysis on the input lowering its dimension from 104 to 86 components. After this step we are left with a model with 59905 degrees of freedom.

Given a NN architecture the model is optimized (trained) minimizing the deviation of the predicted values from the ones computed from first-principles for a set of structures representative of the phenomenon of interest. The creation of such a dataset is detailed in Sec.III. The model error estimate (loss function) is minimized via stochastic gradient descent where mini-batches of the training data are randomly selected and used to compute a noisy estimate of the loss-function gradient and update the model parameters. A portion of the dataset is kept aside for validation on previously unseen data (a standard procedure in ML). Training is achieved when no further improvement is seen on the validation loss. The training procedure is sketched in the right panel of Fig. 1.

\section{CREATION OF THE DATASET}

For empirical potential such as the ones proposed by Tersoff [2] or Stillinger and Weber (SW) [3], one defines a parametric functional form and fixes its few parameters in such a way that a set of stable phases and macroscopic properties are correctly reproduced, assuming that this will allow him/her to perform studies of other related properties. If this is not the case there is not much one can do about. For NN potentials

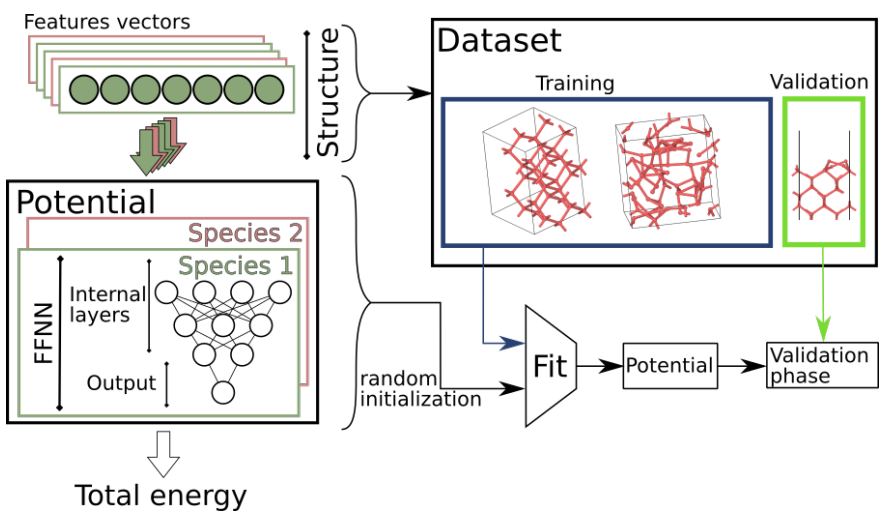

Fig. 1. On the left side: a pictorial view of a FFNN can be realized as an ensemble of nodes arranged in layers. Each node or neuron is a uniquely parametrized mathematical function that takes as input the vector that is passed to the layer it is part of and returns as output the value of the function for the given input. Layers are chained together from the "input" to the "output" in a hierarchical manner such that the output of the above layer is given as input to the next layer. The vector passed to the input layer is commonly defined as the features vector and the output layer is composed of a single neuron the output of which is the local contribution to the total energy. The extrapolation from one FFNN to a potential is trivial: each features vector is labeled with the species of the central atom and is processed accordingly by a different FFNN. The combination of all these networks (one per species) defines a full potential. On the right side: the main training/validation procedure is sketched.

the situation is quite different: once the (great) flexibility of the class of representable functionals is defined by the choice of a specific architecture, the many parameters defining the model are determined in order to reproduce accurate calculation performed on a number or configurations deemed representative. The training/validation dataset is crafted by sampling configurations that will likely be explored during the process of interest. As we are aiming at describing the complex crystal/amorphous interface dynamics in SPE, a number of different phase space regions have been included in the dataset: crystalline and amorphous bulk, surfaces, liquids, and more. The final accuracy in each region depends on the amount of sampling and on the variability of local configurations: regions with limited variability require little sampling while regions with more entropy require larger amounts of data points.

Different parts of the phase space were sampled with different details but all following a common strategy: molecular dynamics (MD) were performed using LAMMPS [10]; from these MD trajectories a number of uncorrelated samples were extracted and their accurate energy and forces were obtained with the Quantum ESPRESSO [11] DFT package (with a cutoff of $50 \mathrm{Ry}$ and a $46.10 \AA$ k-points grid linear-density). These calculated values become part of the dataset. The exchange-correlation functional initially selected was PBE-sol, a PBE-type functional optimized for solid and surfaces [12]. The dataset energies and forces were later recomputed with the original PBE functional [13] to address the unexpected poor description of the melting temperature obtained with PBE-sol (more about this in Results Sec. IV).

Diamond bulk was sampled around its harmonic minimum using Tersoff potential. For other phases, that show higher 
complexity, we proceeded with an iterative scheme similar to the one we proposed in [5]. A first round of MD sampling is performed with empirical or (rough) NN potentials. Points collected from these MDs are used to train new, more accurate, NNs. These potentials are iteratively used to perform more MDs exploring additional, more relevant, geometries to be added in the dataset to improve the quality of the potential. This procedure stops when no appreciable additional phase space sampling is observed in subsequent iterations. Some of the configurations from earlier iterations might then be removed from the dataset as not sampled anymore by the accurate self-consistent potential.

The sampling of the amorphous phase was conducted starting from Tersoff potential and annealing setup similar to one used in previous SPE studies [14]. The liquid was sampled in a similar way as the amorphous. We also added a few solid/liquid interface samples to include realistically intermediate local environments.

Sampling of crystalline surfaces was a bit tricky as none of the commonly used empirical potentials was able to describe correctly the asymmetric dimerization present at the (001) surface. We therefore generated a preliminary NN potential by using part of the database developed by Bartok et al. for their GAP potential [15]. Despite not being optimal for $\mathrm{NN}$ application this database was sufficient to provide a fair starting point reducing the number of self-consistent iterations needed and was kept in the training set for all iterations.

During the evolution, the dataset variability was monitored using a t-sne bidimensional projection of the explored dataset as presented in Fig. 2. This showed how configurations generated by Tersoff and early generation NN potentials explore different geometries from those consistently found by newer generations of $\mathrm{NN}$ potentials.

Approximately 16 thousand points were collected, for a total of 890 thousand local environments. After removing the data from [15], the structures that were no longer visited during the latest iterations of the self consistent procedure, and applying a random uniform sub-sampling on the remaining dataset we were left with approximately 5 thousand data points, or 250 thousand local environments. A standard splitting of the dataset in a training part (containing $80 \%$ of the data) and a validation part (containing $20 \%$ of the data) has been performed, paying attention that the same fraction of training and validation data were present in every subset so as to allow an homogeneous testing in different regions of the phase space.

\section{RESUlts}

The overall validation accuracy of the NN potential trained on the previously described dataset, with energies evaluated according to the PBE exchange-correlation functional, is of $5.6 \mathrm{meV} /$ atom for the energy per atom root mean square error (RMSE) and of $153 \mathrm{meV} / \AA$ mean error per forces component. This accuracy is comparable with the one obtained by others with similar approaches [17]. A more detailed analysis of the validation accuracy decomposed over the different sampled macro regions of the phase space described in the previous

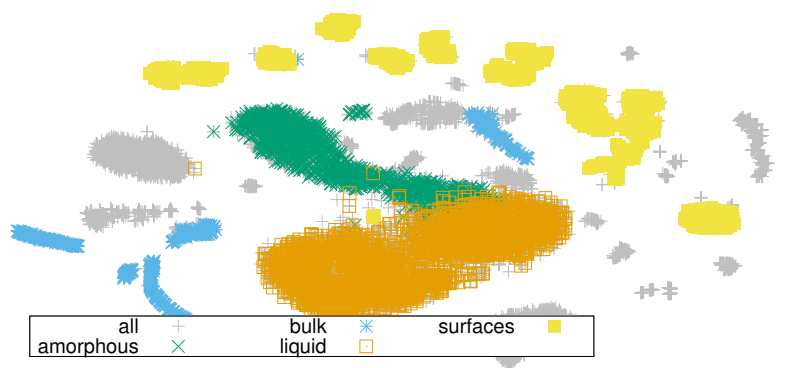

Fig. 2. The figure shows the t-sne applied to the structures in the dataset using the fingerprint defined in Valle et al. [16] and the related distance. In gray all the available structures are presented while colored points refer to the final dataset. Different colors highlight macro-regions of the phase space. Small sparse gray islands are points from the variegate dataset in [15]. Big dense gray islands instead are region sampled by older iterations of the potential excluded because no more visited.

section is shown in Fig. 3 and shows that the accuracy is rather uniform across the phase space.

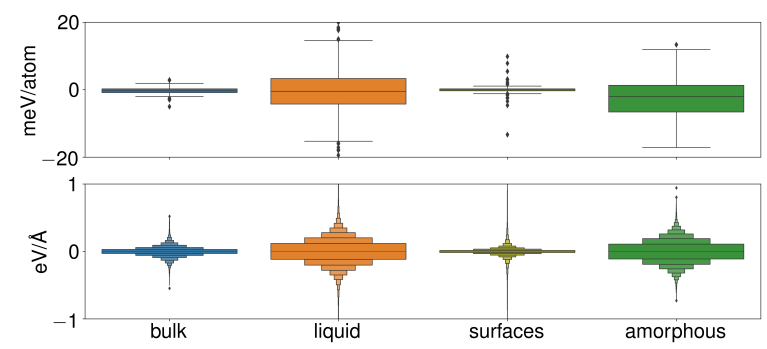

Fig. 3. Distributions of the errors per dataset in energies (top panel, box corresponds to the first quartile) and Forces (bottom panel, different boxes represent different quartiles) are presented. It can be seen how phases with the highest variability (liquid and amorphous) show the highest errors. At this point different RMSE can be computed for energies and forces depending on the subset of points: $5.8 \mathrm{meV}-170 \mathrm{meV} / \AA$ for the amorphous, $0.9 \mathrm{meV}$ $66 \mathrm{meV} / \AA$ for the bulk, $7.5 \mathrm{meV}-196 \mathrm{meV} / \AA$ for the liquid and $3.4 \mathrm{meV}$ $103 \mathrm{meV} / \AA$ for the surfaces.

In order to get a feeling of the quality of the potential associated to these reported numerical errors we tested it investigating a few physical properties that can be compared with experiments and/or DFT references. A comprehensive test has been carried out for the diamond structure where we checked the accuracy of the Energy-Volume curve and computed the bulk modulus obtaining a value of $98.0 \mathrm{GPa}$, close to the experimental value of $97.6 \mathrm{GPa}$ [18]. Full phonon dispersions were also investigated obtaining results very close to the DFT reference (see bottom panel of Fig. 4).

SPE dynamics critically depends on the temperature involved and commonly adopted empirical potentials either fail terribly in describing the thermal stability of the different phases or, if they succeed in it, fail in other aspects.

For example, the SW potential has a melting temperature of $1691 \pm 20 \mathrm{~K}$ [19] but generates amorphous of poor quality. Tersoff potential, on the other hand, is believed to generate realistic amorphous geometries but it predicts a melting tem- 
perature of $2547 \pm 22 \mathrm{~K}$ [20]. Errors in the predicted melting temperature are likely to impact defect formation and diffusion dynamics, relevant for SPE. We hence turned to the study of the bulk melting temperature, equilibrating samples where crystalline and liquid phases coexist [21]-[23].

We were disappointed to find out that the PBE-sol functional originally adopted gave a NN potential whose bulk melting temperature was as low as $1194 \pm 28 \mathrm{~K}$. This induced us, after several cross checks, to resort to the original PBE xcfunctional, obtaining a melting temperature of $1468 \pm 11 \mathrm{~K}$, in line with the literature value of $1540 \pm 50 \mathrm{~K}$ [24], still slightly off w.r.t. the experimental value of $1685 \pm 2 \mathrm{~K}$. The unsuitability of PBE-sol functional to describe melting behavior of materials has been recently independently confirmed for GAP potentials [25].

Having obtained a reasonable description of the liquid/solid transition we proceeded to study the amorphous phase. We followed the procedure described in Ref. [26], [27] where liquid samples were quenched with a 10K/ps ramp. Amorphous samples of very good quality are thus obtained. In the top panel of Fig. 4 the structure factor from the largest simulated cell (4096 atoms) is compared with experimental results.
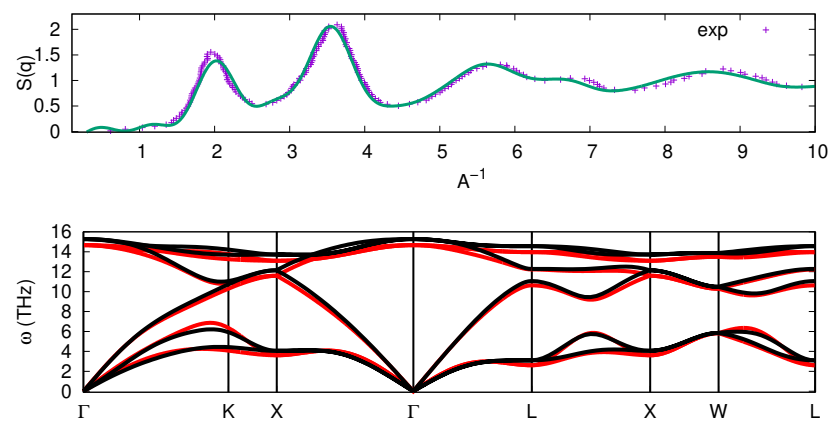

Fig. 4. Top Panel: Amorphous silicon structure factor $S(q)$. Dots are experimental points [28], green line from NN potential. Bottom Panel: Diamond phonons of silicon. DFT prediction with PBE (black line) and NN (red line).

Next we examined in detail the (001) surfaces that play a very important role on microelectronic devices [29]. Several reconstructions have been proposed in [29]: $\mathrm{p}(1 \mathrm{x} 1)$ also known as Ideal, $\mathrm{p}(2 \times 1)$ symmetric, $\mathrm{p}(2 \times 1)$ asymmetric, $\mathrm{p}(2 \times 2)$ and $\mathrm{c}(4 \times 2)$. Of these four structures only the last three have proven to be stable with sufficiently accurate calculations. The energy differences among predicted structures can be seen in Fig. 5. Geometries were also compared and our NN potential has shown to be able to correctly predict the dimer tilting angle of $18^{\circ}$ for the $\mathrm{p}(2 \times 1)$ asymmetric phase as well as the $19^{\circ}$ angle of the $\mathrm{p}(2 \times 2)$ and $\mathrm{c}(4 \times 2)$ ones, in accordance with DFT.

\section{Preliminary observation of the SPE mechanisms}

The overall broad accuracy shown by our NN potential for so many properties gives us confidence in its adequacy to explore the more complex phenomenon of SPE. Using a MD procedure similar to the one described in [27], we created an amorphous/crystalline diamond interface and performed a first

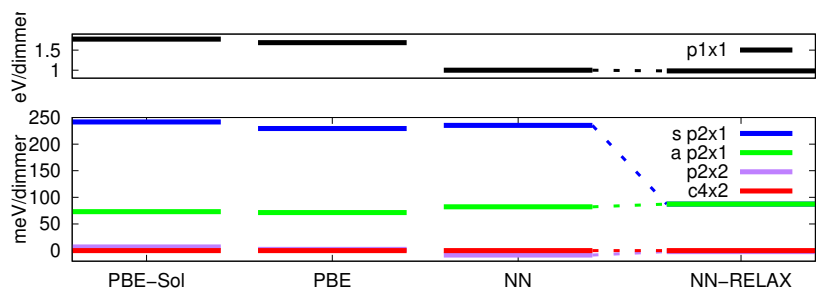

Fig. 5. Surfaces energies. From left to right, PBE-Sol energies, PBE energies, $\mathrm{NN}$ energies for PBE geometries, NN energies after relaxation. The $\mathrm{c}(4 \times 2)$ structure has always been taken as zero reference. DFT geometries have been obtained by relaxation with the BFGS algorithm, as a force threshold we opted for the default $25 \mathrm{meV} / \AA$. Minimization with $\mathrm{NN}$ potentials were performed with the same threshold. Both DFT calculations predicted the $\mathrm{p}(2 \times 1)$ symmetric structure as stable with a low k-points grid, the reported energy is a successive SCF calculation with the high accuracy grid. In the last column the $\mathrm{s} \mathrm{p}(2 \times 1)$ geometry has relaxed to the a $\mathrm{p}(2 \times 1)$ structure. The $\mathrm{p}(2 \times 2)$ and the $\mathrm{c}(4 \times 2)$ are incorrectly ordered but their difference in energy is below our error threshold.

SPE simulation at $1050 \mathrm{~K}$. Already in this preliminary simulation we can see that 3D pyramidal motives, expected from the different reconstructions speed measured experimentally [30], appear (presented in Fig. 6). A detailed study of SPE mechanism and speed is underway.

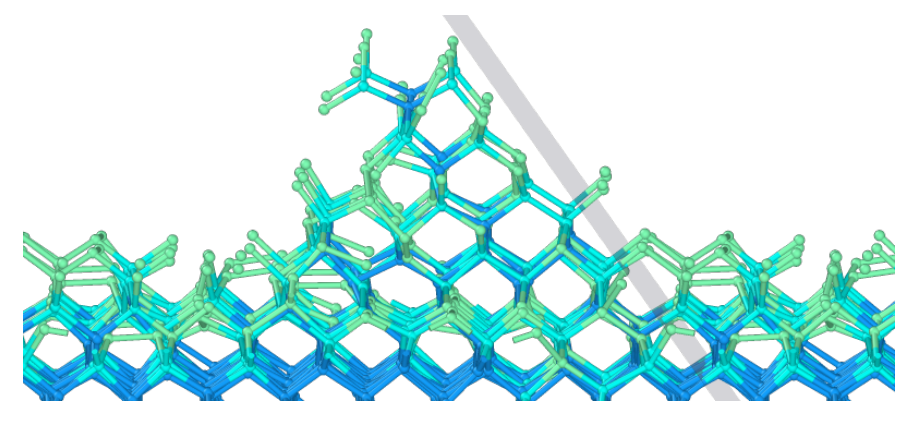

Fig. 6. 3D pyramidal motives appearing during the solid phase epitaxy simulation at $1050 \mathrm{~K}$. Only tetrahedrally coordinated atoms with angles compatible with the diamond structure are presented for simplicity.

\section{CONCLUSiOnS AND OUTLOOK}

We have shown that $\mathrm{NN}$ potentials are a valid way to model physical phenomena at the atomistic level through the creation and testing of a potential for silicon from a diverse dataset, crafted from crystalline, amorphous, liquid and surface configurations. As a byproduct, we found that PBE-sol xc-functional is not suitable to study thermal related phenomena in silicon. With the developed potential we are investigating the SPE process, focusing on the underlying recrystallization mechanism and speed. We expect that the resulting insight will allow us to improve the prediction of TCAD codes, possibly solving the longstanding problem of the experimentally observed difference in recrystallization speed in different growth directions.

\section{ACKNOWLEDGEMENTS}

We acknowledge funding from European Union Horizon 2020 research and innovation program under grant agreement No. 871813 MUNDFAB and No. 824143 MaX CoE. 


\section{REFERENCES}

[1] N. Zographos, C. Zechner, I. Martin-Bragado, K. Lee, and Y.-S. Oh, "Multiscale modeling of doping processes in advanced semiconductor devices," Materials Science in Semiconductor Processing, vol. 62, pp. 49-61, may 2017.

[2] J. Tersoff, "New empirical model for the structural properties of silicon," Phys. Rev. Lett., vol. 56, pp. 632-635, Feb. 1986.

[3] F. H. Stillinger and T. A. Weber, "Computer simulation of local order in condensed phases of silicon," Phys. Rev. B, vol. 31, pp. 5262-5271, Apr. 1985.

[4] R. Lot, F. Pellegrini, Y. Shaidu, and E. Küçükbenli, "PANNA: Properties from artificial neural network architectures," Comput. Phys. Commun., vol. 256, p. 107402, Nov. 2020.

[5] Y. Shaidu, E. Küçükbenli, R. Lot, F. Pellegrini, E. Kaxiras, and S. de Gironcoli, "A systematic approach to generating accurate neural network potentials: The case of carbon," npj Comput Mater, vol. 7, Apr. 2021.

[6] J. Smith, O. Isayev, and A. Roitberg, "ANI-1: An extensible neural network potential with DFT accuracy at force field computational cost," Chem. Sci., vol. 8, no. 4, pp. 3192-3203, 2017.

[7] J. Behler, "Neural network potential-energy surfaces in chemistry: a tool for large-scale simulations," Physical Chemistry Chemical Physics, vol. 13, no. 40, p. 17930, 2011.

[8] J. Behler and M. Parrinello, "Generalized neural-network representation of high-dimensional potential-energy surfaces," Phys. Rev. Lett., vol. 98, Apr. 2007.

[9] Y. Zuo, C. Chen, X. Li, Z. Deng, Y. Chen, J. Behler, G. Csányi, A. V. Shapeev, A. P. Thompson, M. A. Wood, and S. P. Ong, "Performance and cost assessment of machine learning interatomic potentials," J. Phys. Chem. A, vol. 124, pp. 731-745, Jan. 2020.

[10] S. Plimpton, "Fast parallel algorithms for short-range molecular dynamics," Journal of Computational Physics, vol. 117, pp. 1-19, mar 1995.

[11] P. Giannozzi, O. Baseggio, P. Bonfà, D. Brunato, R. Car, I. Carnimeo, C. Cavazzoni, S. de Gironcoli, P. Delugas, F. Ferrari Ruffino, A. Ferretti, N. Marzari, I. Timrov, A. Urru, and S. Baroni, "QuantumESPRESSO toward the exascale," J. Chem. Phys., vol. 152, p. 154105, Apr. 2020.

[12] J. P. Perdew, A. Ruzsinszky, G. I. Csonka, O. A. Vydrov, G. E. Scuseria, L. A. Constantin, X. Zhou, and K. Burke, "Restoring the density-gradient expansion for exchange in solids and surfaces," Physical Review Letters, vol. 100, apr 2008.

[13] J. P. Perdew, K. Burke, and M. Ernzerhof, "Generalized gradient approximation made simple," Physical Review Letters, vol. 77, pp. 38653868 , oct 1996 .

[14] C. Krzeminski, Q. Brulin, V. Cuny, E. Lecat, E. Lampin, and F. Cleri, "Molecular dynamics simulation of the recrystallization of amorphous Si layers: Comprehensive study of the dependence of the recrystallization velocity on the interatomic potential," J. Appl. Phys., vol. 101, p. 123506 June 2007.

[15] A. P. Bartók, J. Kermode, N. Bernstein, and G. Csányi, "Machine learning a general-purpose interatomic potential for silicon," Phys. Rev. $X$, vol. 8, Dec. 2018.

[16] M. Valle and A. R. Oganov, "Crystal fingerprint space - a novel paradigm for studying crystal-structure sets," Acta Cryst Sect A, vol. 66, pp. 507-517, Aug. 2010.

[17] N. Artrith and J. Behler, "High-dimensional neural network potentials for metal surfaces: A prototype study for copper,' Phys. Rev. B, vol. 85, Jan. 2012.

[18] M. A. Hopcroft, W. D. Nix, and T. W. Kenny, "What is the young's modulus of silicon?," Journal of Microelectromechanical Systems, vol. 19 no. 2, pp. 229-238, 2010

[19] J. Broughton and X. Li, "Phase diagram of silicon by molecular dynamics," Phys. Rev. B, vol. 35, pp. 9120-9127, June 1987.

[20] S. J. Cook and P. Clancy, "Comparison of semi-empirical potential functions for silicon and germanium," Phys. Rev. B, vol. 47, pp. 76867699, Apr. 1993.

[21] Y. Zhang and E. J. Maginn, "A comparison of methods for melting point calculation using molecular dynamics simulations," J. Chem. Phys., vol. 136, p. 144116, Apr. 2012.

[22] J. Morris, C. Wang, K. Ho, and C. Chan, "Melting line of aluminum from simulations of coexisting phases," Phys. Rev. B, vol. 49, pp. 31093115, Feb. 1994.
[23] J. R. Morris and X. Song, "The melting lines of model systems calculated from coexistence simulations," J. Chem. Phys., vol. 116, pp. 9352-9358, June 2002.

[24] S. Yoo, S. S. Xantheas, and X. C. Zeng, "The melting temperature of bulk silicon from ab initio molecular dynamics simulations," Chem. Phys. Lett., vol. 481, pp. 88-90, Oct. 2009.

[25] R. Jinnouchi, F. Karsai, and G. Kresse, "On-the-fly machine learning force field generation: Application to melting points," Physical Review $B$, vol. 100, jul 2019.

[26] K. Laaziri, S. Kycia, S. Roorda, M. Chicoine, J. Robertson, J. Wang, and S. Moss, "High-energy x-ray diffraction study of pure amorphous silicon," Phys. Rev. B, vol. 60, pp. 13520-13533, Nov. 1999.

[27] E. Lampin and C. Krzeminski, "Molecular dynamics simulations of the solid phase epitaxy of si: Growth mechanism and orientation effects," J. Appl. Phys., vol. 106, p. 063519, Sept. 2009.

[28] K. Laaziri, S. Kycia, S. Roorda, M. Chicoine, J. Robertson, J. Wang, and S. Moss, "High resolution radial distribution function of pure amorphous silicon," Phys. Rev. Lett., vol. 82, pp. 3460-3463, Apr. 1999.

[29] A. Ramstad, G. Brocks, and P. Kelly, "Theoretical study of the si(100) surface reconstruction," Phys. Rev. B, vol. 51, pp. 14504-14523, May 1995.

[30] G. Olson and J. Roth, "Kinetics of solid phase crystallization in amorphous silicon," Mater. Sci. Rep., vol. 3, pp. 1-77, Jan. 1988. 